\title{
$\mathrm{N}_{2} \mathrm{O}$ formation mechanism during low-
}

\section{temperature $\mathrm{NH}_{3}-\mathrm{SCR}$ over $\mathrm{Cu}-\mathrm{SSZ}-13$}

\section{catalysts with different $\mathrm{Cu}$ loadings}

Dongwei Yao ${ }^{a . \#,{ }^{*}, \text { Biao Liu }}{ }^{a, \#}$, Feng $W u^{a,{ }^{*},}$ Yuxi Li ${ }^{a}$, Xiaohan Hu ${ }^{a}$, Weiyang Jin ${ }^{b}$, Xinlei Wang $^{c}$

(a College of Energy Engineering, Zhejiang University, Hangzhou 310027, China

b Wuxi Weifu Environmental Catalysts Co., Ltd, Wuxi 214018, China

c Department of Agricultural and Biological Engineering, University of Illinois at Urbana-Champaign, Urbana 61801, USA)

* Corresponding author at: College of Energy Engineering, Zhejiang University, 38 Zheda Road, West Lake District, Hangzhou, Zhejiang Province 310027, China

E-mail: dwyao@zju.edu.cn (Dongwei Yao); wfice@zju.edu.cn (Feng Wu)

\# These authors are co-first authors of the article 


\section{EPR}
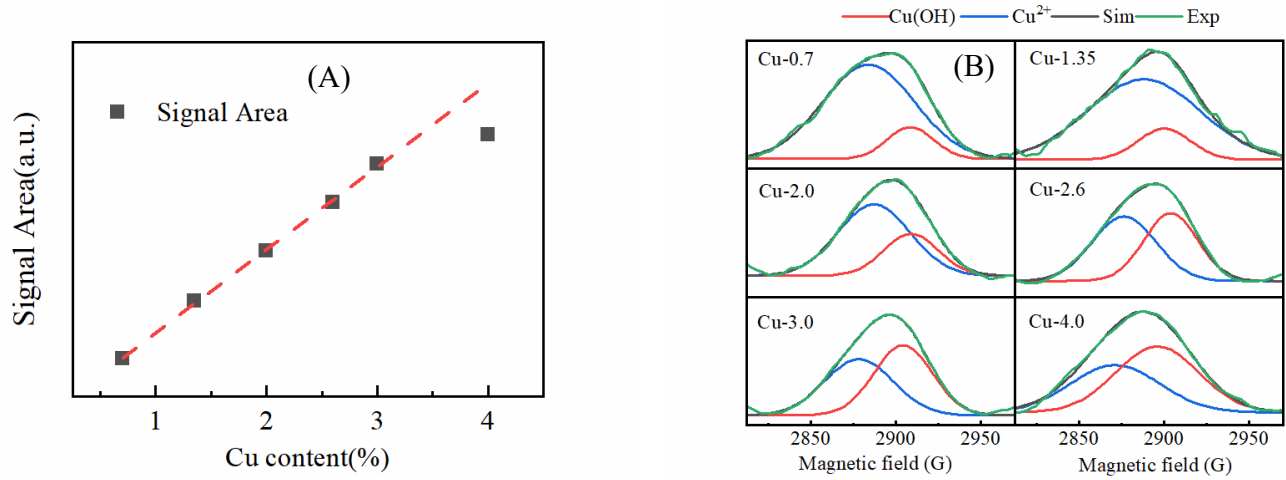

Figure $\mathrm{S} 1$ (A) Amount of $\mathrm{Cu}^{2+}$ ions quantified by double integrating the EPR spectra, (B) Deconvolution of EPR spectra over Cu-SSZ-13 catalysts

Table $\mathrm{S} 1 \mathrm{The}$ relative ratios of $\mathrm{Cu}^{2+},[\mathrm{Cu}(\mathrm{OH})]^{+}$and $\mathrm{CuO}$ species based on EPR analysis

\begin{tabular}{cccc}
\hline Catalysts & $\mathrm{Cu}^{2+}(\%)$ & {$[\mathrm{Cu}(\mathrm{OH})]^{+}(\%)$} & $\mathrm{CuO}(\%)$ \\
\hline $\mathrm{Cu}-0$ & 0 & 0 & 0 \\
$\mathrm{Cu}-0.7$ & 85.39 & 14.61 & 0 \\
$\mathrm{Cu}-1.35$ & 84.69 & 15.31 & 0 \\
$\mathrm{Cu}-2.0$ & 69.25 & 30.75 & 0 \\
$\mathrm{Cu}-2.6$ & 53.13 & 46.87 & 0 \\
$\mathrm{Cu}-3.0$ & 48.55 & 51.45 & 0 \\
$\mathrm{Cu}-4.0$ & 36.18 & 47.41 & 16.41 \\
\hline
\end{tabular}




\section{2. $\mathrm{H}_{2}$-TPR}

The relative ratios of $\mathrm{Cu} 2+,[\mathrm{Cu}(\mathrm{OH})]+$ and $\mathrm{CuO}$ species based on TPR analysis are shown in Table S1.

Table $\mathrm{S} 2 \mathrm{The}$ relative ratios of $\mathrm{Cu}^{2+},[\mathrm{Cu}(\mathrm{OH})]^{+}$and $\mathrm{CuO}$ species based on TPR analysis

\begin{tabular}{cccc}
\hline Catalysts & $\mathrm{Cu}^{2+}(\%)$ & {$[\mathrm{Cu}(\mathrm{OH})]^{+}(\%)$} & $\mathrm{CuO}(\%)$ \\
\hline $\mathrm{Cu}-0$ & 0 & 0 & 0 \\
$\mathrm{Cu}-0.7$ & 83.65 & 16.35 & 0 \\
$\mathrm{Cu}-1.35$ & 82.65 & 17.35 & 0 \\
$\mathrm{Cu}-2.0$ & 66.12 & 33.88 & 0 \\
$\mathrm{Cu}-2.6$ & 53.64 & 44.36 & 0 \\
$\mathrm{Cu}-3.0$ & 45.16 & 54.84 & 0 \\
$\mathrm{Cu}-4.0$ & 36.18 & 49.14 & 14.68 \\
\hline
\end{tabular}

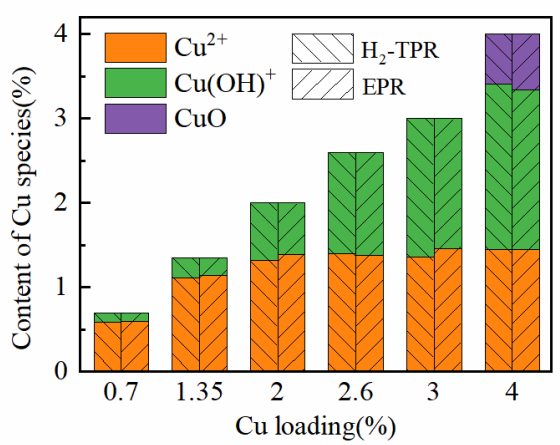

Figure S2 The amount of $\mathrm{Cu}^{2+},[\mathrm{Cu}(\mathrm{OH})]^{+}$and $\mathrm{CuO}$ species over $\mathrm{Cu}-\mathrm{SSZ}-13$ catalysts with different $\mathrm{Cu}$ contents calculated by $\mathrm{H}_{2}$-TPR and EPR

\section{NOx conversion}

$\mathrm{NOx}$ and $\mathrm{NH}_{3}$ conversion are calculated using the equations shown as below:

$$
\begin{aligned}
& \text { NOx conversion }=\left(1-\left[\mathrm{NO}_{\mathrm{X}}\right]_{\text {outlet }} /\left[\mathrm{NO}_{\mathrm{X}}\right]_{\text {inlet }}\right) \times 100 \% \\
& \mathrm{NH}_{3} \text { conversion }=\left(1-\left[\mathrm{NH}_{3}\right]_{\text {outlet }} /\left[\mathrm{NH}_{3}\right]_{\text {inlet }}\right) \times 100 \%
\end{aligned}
$$




$$
\mathrm{N}_{2} \text { selectivity }=\left(1-\frac{2\left[\mathrm{~N}_{2} \mathrm{O}\right]_{\text {out }}}{[\mathrm{NOx}]_{i n}+\left[\mathrm{NH}_{3}\right]_{\text {in }}}\right) \times 100 \%
$$
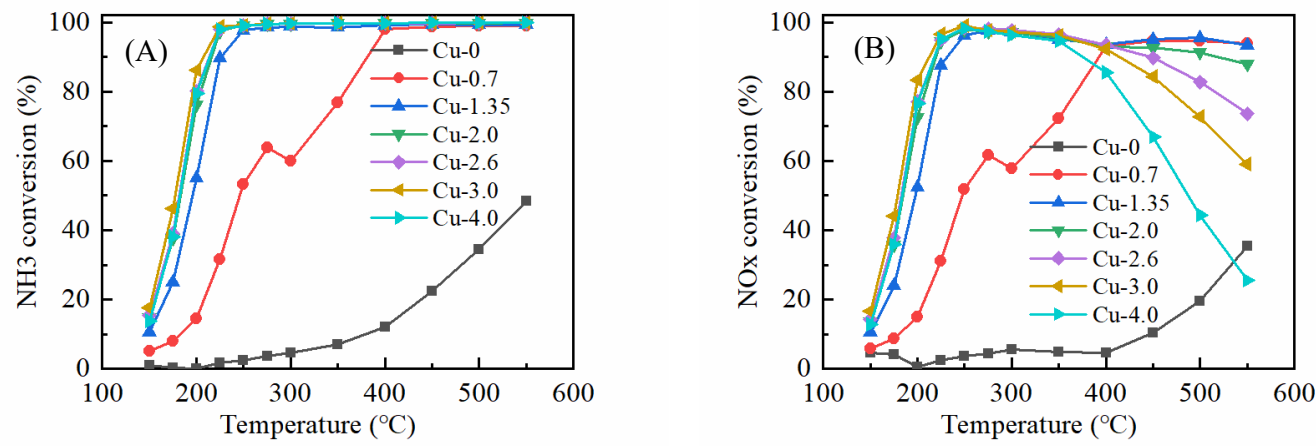

Figure $\mathrm{S} 3$ (A) $\mathrm{NH}_{3}$ and (B) NOx conversion under standard SCR conditions
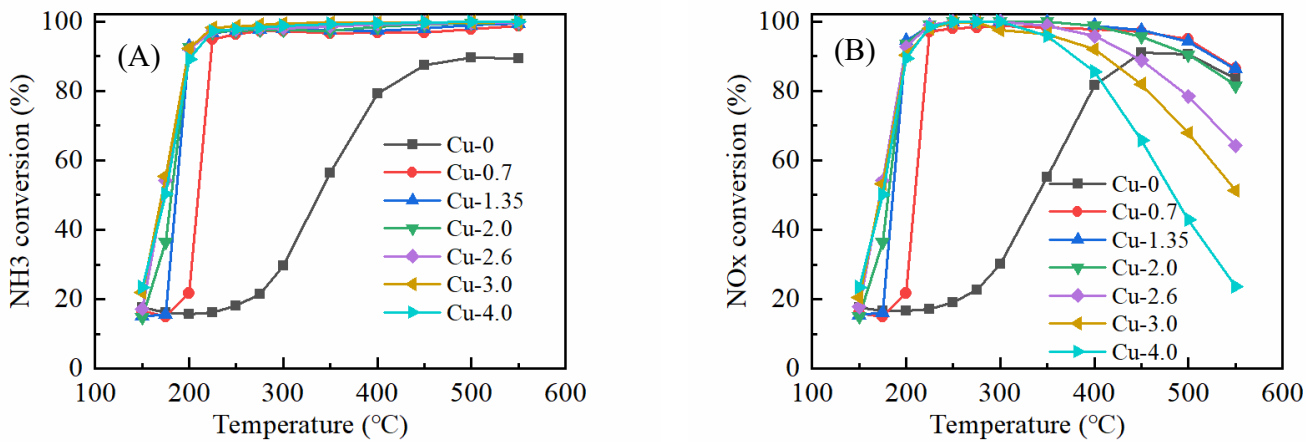

Figure S4 (A) $\mathrm{NH}_{3}$ and (B) NOx conversion under fast SCR conditions
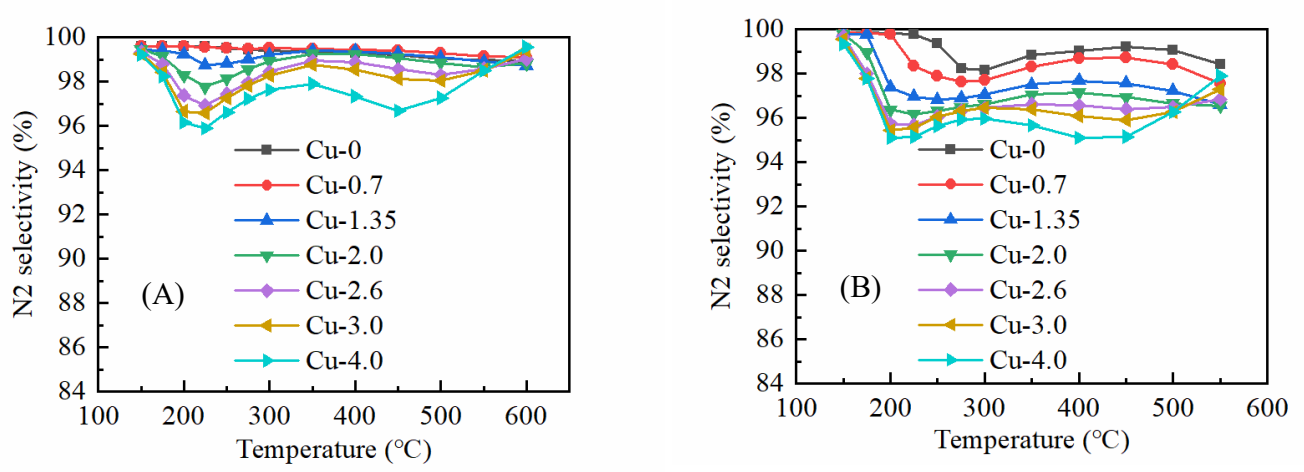

Figure S5 $\mathrm{N}_{2}$ selectivity under (A) standard and (B) fast SCR conditions 


\section{Oxidation}
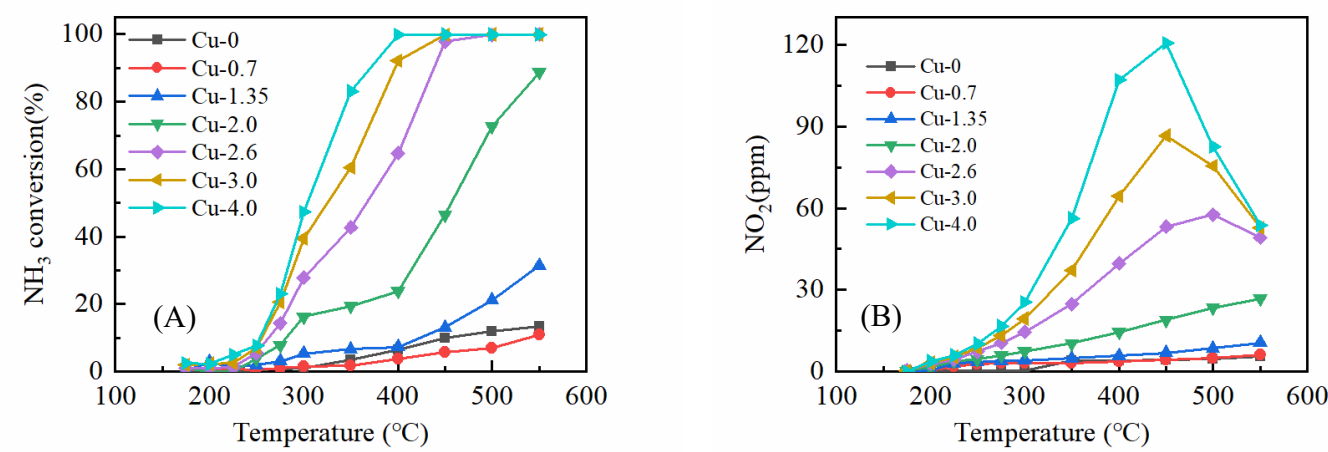

Figure $\mathrm{S} 6$ (A) $\mathrm{NH}_{3}$ conversion under $\mathrm{NH}_{3}$ oxidation condition and (B) $\mathrm{NO}_{2}$ formation under NO oxidation condition

\section{DRIFTS}

\section{$5.1 \mathrm{NH}_{3}$ adsorption}
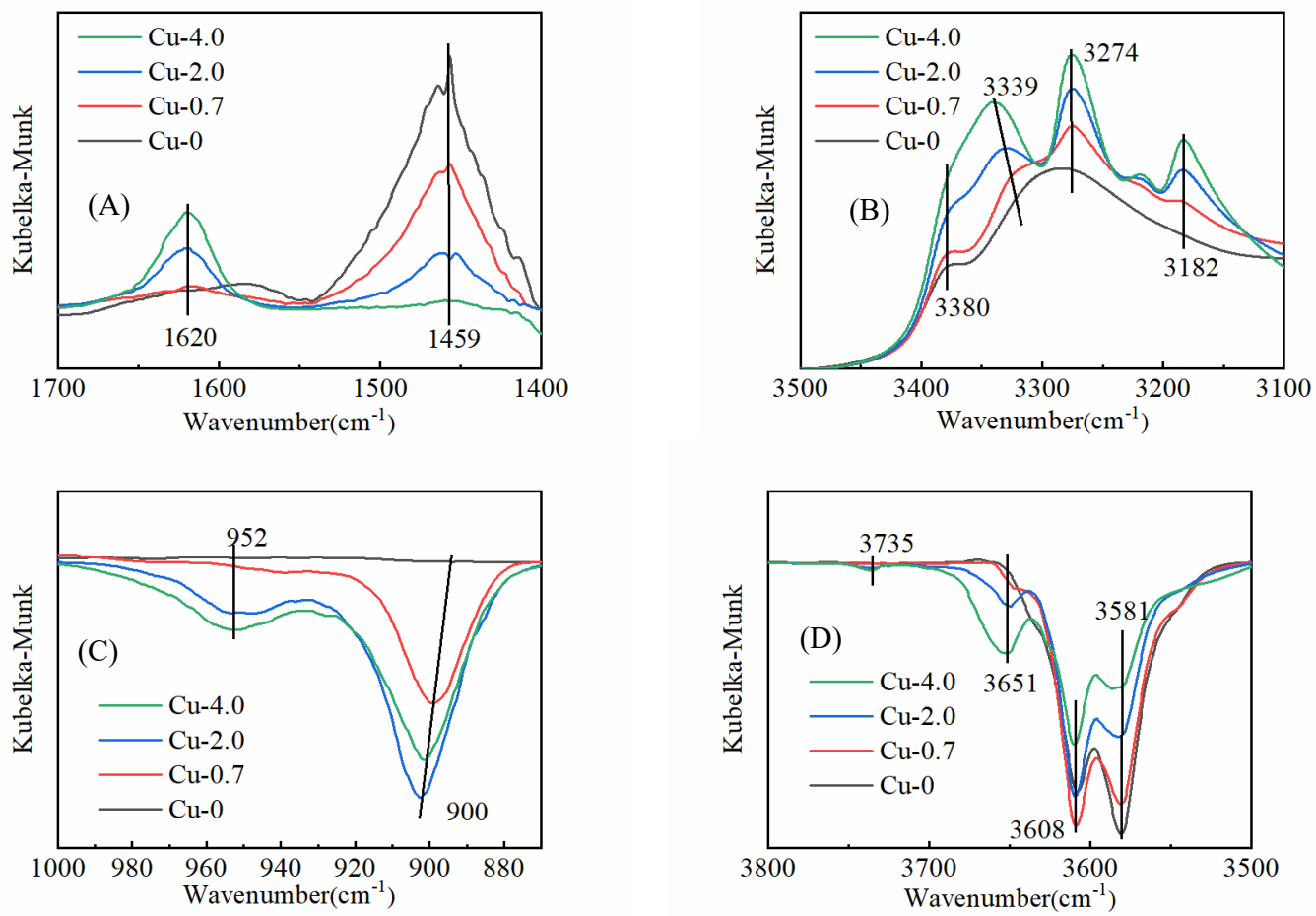

Figure S7 (A) $1700-1400 \mathrm{~cm}^{-1}$, (B) $3500-3100 \mathrm{~cm}^{-1}$, (C) $1000-870 \mathrm{~cm}^{-1}$ and (D) 3800$3500 \mathrm{~cm}^{-1}$ at $150^{\circ} \mathrm{C}$ after $\mathrm{NH}_{3}$ adsorption over different catalysts 


\section{$5.2 \mathrm{NO}+\mathrm{O}_{2}$ react with pre-adsorbed $\mathrm{NH}_{3}$}
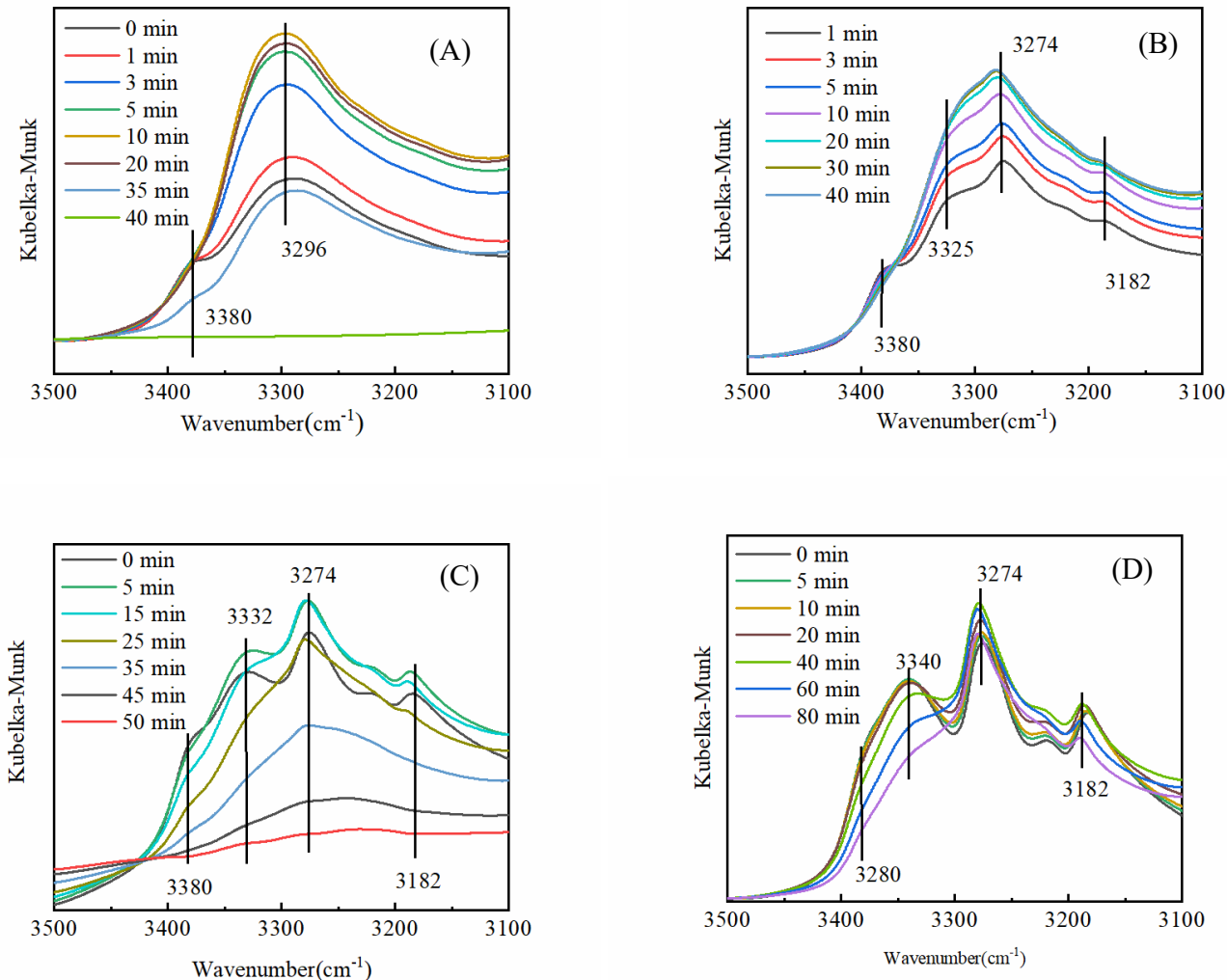

Figure S8 DRIFTS spectra obtained (64 scans) in the range between $3500-3100 \mathrm{~cm}^{-1}$ upon $\mathrm{NO}+\mathrm{O}_{2}$ passing over (A) $\mathrm{Cu}-0,(\mathrm{~B}) \mathrm{Cu}-0.7,(\mathrm{C}) \mathrm{Cu}-2.0$ and (D) $\mathrm{Cu}-4.0$ catalysts with pre-adsorbed $\mathrm{NH}_{3}$ 


\section{$5.3 \mathrm{NO}_{2}$ react with pre-adsorbed $\mathrm{NH}_{3}$}
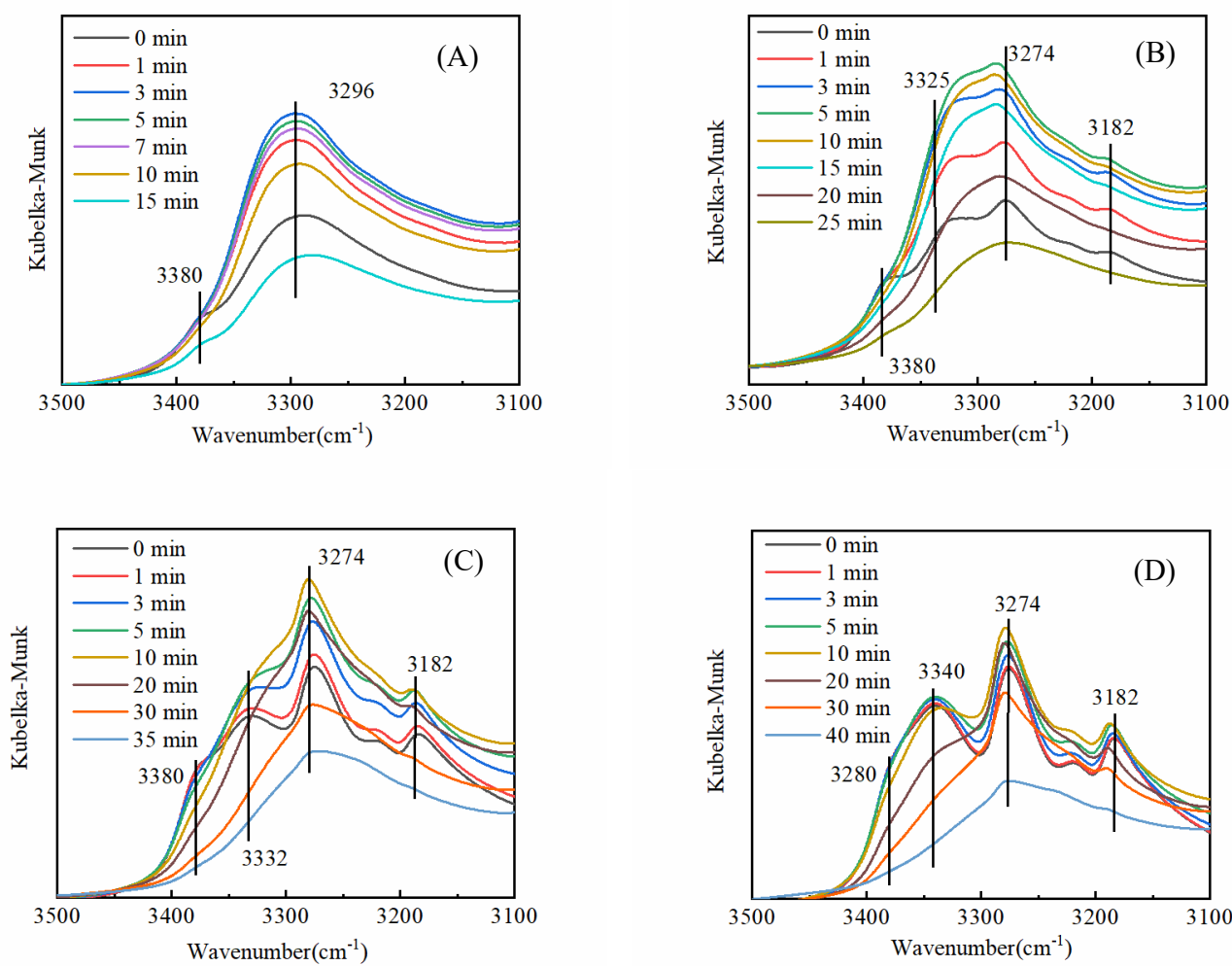

Figure S9 DRIFTS spectra obtained (64 scans) in the range between $3500-3100 \mathrm{~cm}^{-1}$ upon $\mathrm{NO}_{2}$ passing over (A) $\mathrm{Cu}-0$, (B) $\mathrm{Cu}-0.7$, (C) $\mathrm{Cu}-2.0$ and (D) $\mathrm{Cu}-4.0$ catalysts with pre-adsorbed $\mathrm{NH}_{3}$ 


\subsection{NOx adsorption}
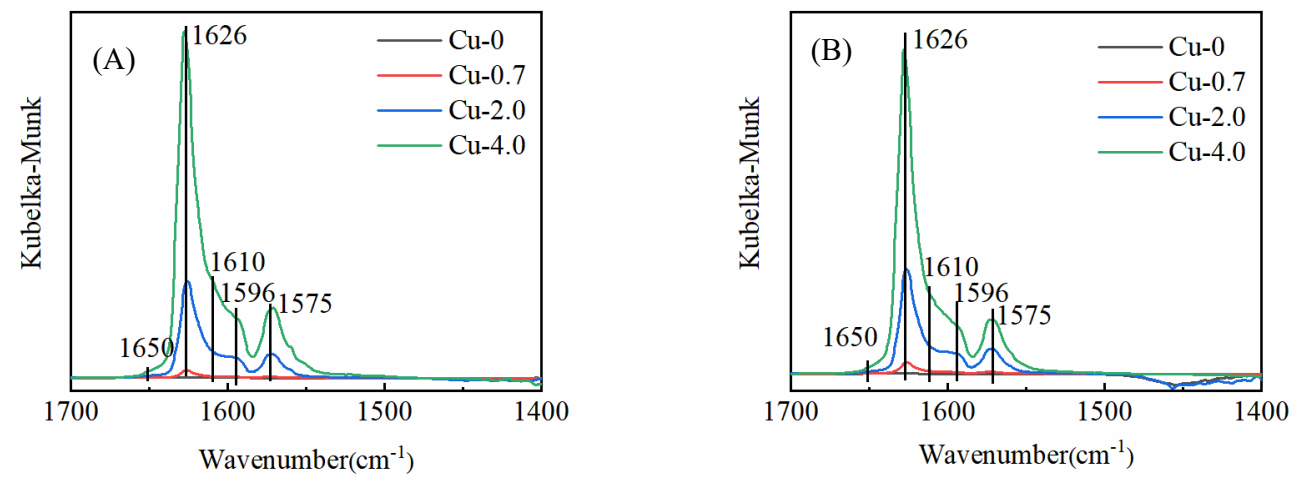

Figure S10 DRIFTS spectra obtained between 1700-1400 $\mathrm{cm}^{-1}$ after (A) $\mathrm{NO}+\mathrm{O}_{2}$ and (B) $\mathrm{NO}_{2}$ adsorption at $150^{\circ} \mathrm{C}$ over different catalysts
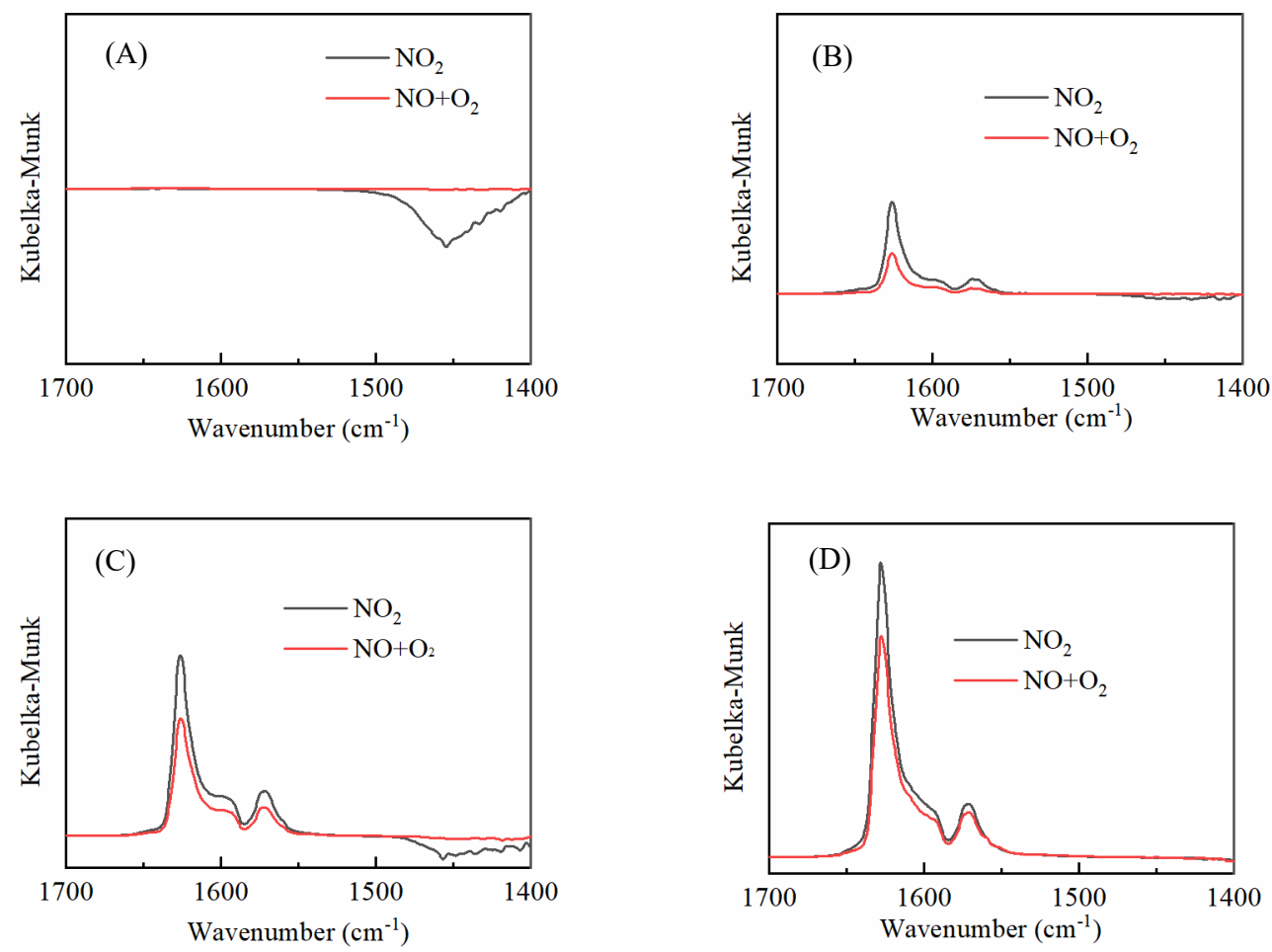

Figure $\mathrm{S} 11$ A compare between $\mathrm{NO}+\mathrm{O}_{2}$ and $\mathrm{NO}_{2}$ adsorption over (A) $\mathrm{Cu}-0$, (B) $\mathrm{Cu}-$ 0.7, (C) Cu-2.0 and (D) Cu-4.0 samples 
5.5 Compare of $\mathrm{NH}_{3}$ adsorption, $\mathrm{NH}_{3}$ react with pre-adsorbed $\mathrm{NO}+\mathrm{O}_{2}$ and $\mathrm{NO}_{2}$
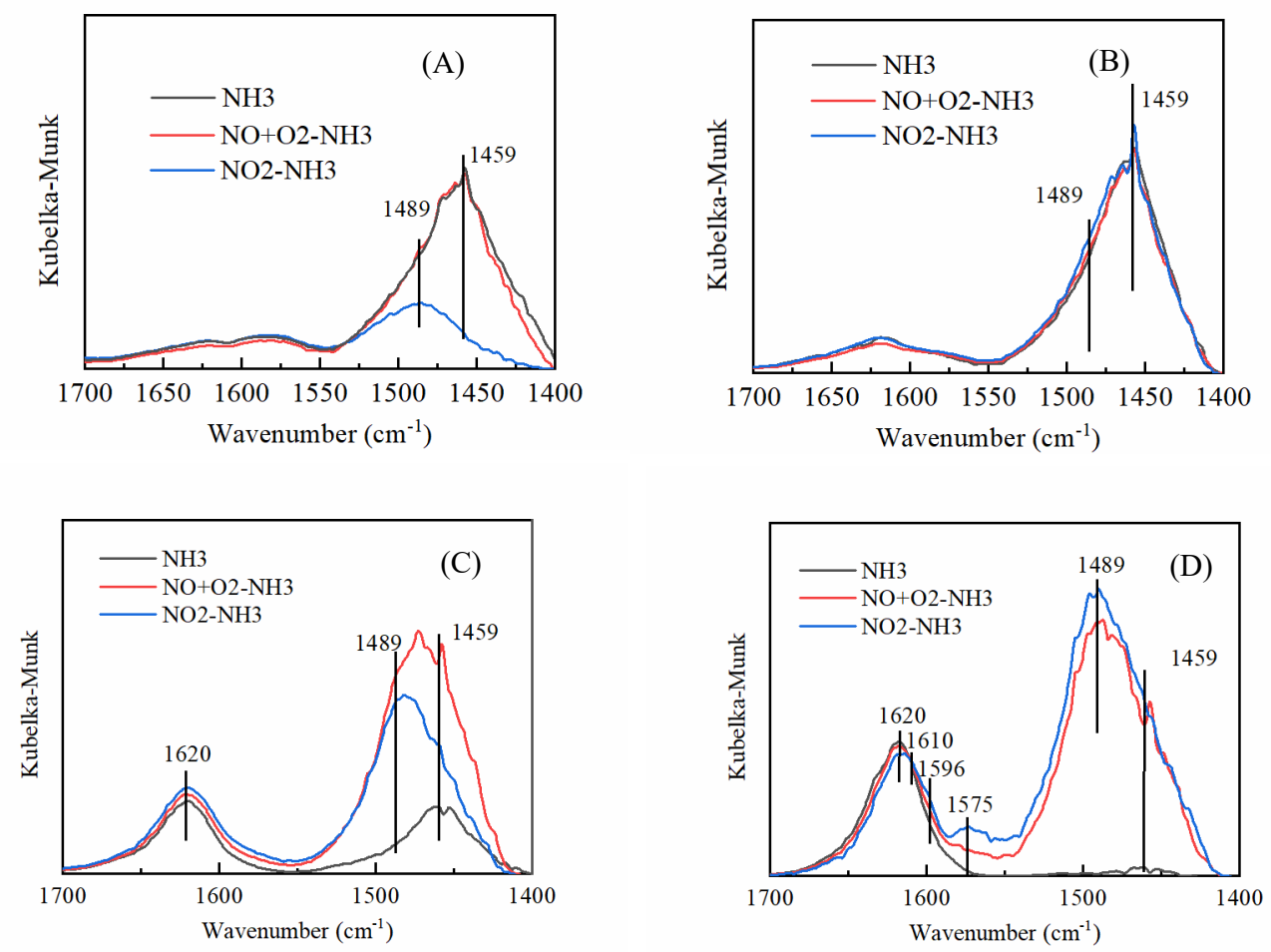

Figure S12 DRIFTS spectra obtained (64 scans) in the range between $1700-1400 \mathrm{~cm}^{-}$

${ }_{1}^{1}$ after $\mathrm{NH}_{3}$ adsorption, $\mathrm{NH}_{3}$ react with pre-adsorbed $\mathrm{NO}+\mathrm{O}_{2}$ and $\mathrm{NO}_{2}$ over $(\mathrm{A}) \mathrm{Cu}-$ 0, (B) $\mathrm{Cu}-0.7,(\mathrm{C}) \mathrm{Cu}-2.0$ and (D) $\mathrm{Cu}-4.0$ catalysts 